\title{
Two-Settlement Electricity Markets with Price Caps and Cournot Generation Firms
}

\author{
Jian Yao, Shmuel S. Oren, Ilan Adler \\ Department of Industrial Engineering and Operations Research \\ 4141 Etcheverry Hall, University of California at Berkeley \\ Berkeley, CA 94720, US \\ \{jyao, oren, adler\}@ieor.berkeley.edu
}

June 2005

We compare two alternative mechanisms for capping prices in two-settlement electricity markets. With sufficient lead time, forward market prices are implicitly capped by competitive pressure of potential entry that will occur when forward prices rise above some backstop price. Another more direct approach is to cap spot prices through a performance-based regulatory intervention. In this paper we explore the implications of these two alternative mechanisms in a two-settlement Cournot equilibrium framework. We formulate the market equilibrium as a stochastic equilibrium problem with equilibrium constraints (EPEC) capturing congestion effects, probabilistic contingencies and horizontal market power. As an illustrative test case, we use the 53-bus Belgian electricity network with representative generator costs but hypothetical demand and ownership structure. Compared to a price-uncapped two-settlement system, a forward cap increases firms' incentives for forward contracting, whereas a spot cap reduces such incentives. Moreover, in both cases, more forward contracts are committed as the generation resource ownership structure becomes more diversified. keyword: OR in energy, Electricity Markets, Cournot Equilibrium, Mathematical Program with Equilibrium Constraints, Equilibrium Problem with Equilibrium Constraints.

\section{Introduction}

It is generally agreed that forward contracting mitigates generators' horizontal market power in the spot market and protects market participants against spot price volatility resulting from system contingencies and demand uncertainty. Previous contributions, such as $[1,2,12,19]$, focus on the impact of forward markets on spot prices and social welfare under alternative assumptions regarding the relationship between forward and spot prices. It is shown that generators have incentives to trade in the forward markets whereas forward contracting reduces spot prices and increases consumption levels and social welfare. These models assume a fixed generation stock which is appropriate for two-settlement system over short time intervals (e.g., day ahead and real time markets). Competitive entry in the forward market and regulatory caps on spot prices are further means of mitigating price spikes and market power abuse. For long-term forward contracts,

\footnotetext{
${ }^{1}$ Research supported by the National Science Foundation Grant ECS-0224779 and by the Power System Engineering Research Center (PSERC). The authors wish to thank Dr. Bert Willems who provided the network data for the Belgian electricity system used in this paper.

${ }^{2}$ A preliminary abridged version of this paper appeared in the Proceeding of the 38th Hawaii International Conference on Systems Sciences (HICCS38), Jan 4-7, 2005.
} 
potential competitive entry imposes an implicit price cap on forward contract prices since new investment in generation capacity will occur when forward prices rise above the amortized long-run generation cost. Alternatively, regulators in many restructured electricity markets have imposed price or bid caps in the spot markets in an attempt to rectify market imperfections such as demand inelasticity, barriers to entry, imperfect capital markets and locational market power. In this paper we extend our earlier model in [19] by considering, separately, the effects of these two cap types on the spot and forward prices.

In particular, we address the following questions. To what extent do the generators commit forward contracts under price caps? How do caps affect the spot and forward prices? What is the relative impact of spot caps versus competitive entry in mitigating market power? How sensitive is the total forward commitment with respect to the resource ownership structure and the cap levels? We study these questions via a two-settlement Cournot equilibrium model where generation firms have horizontal market power. The system is subject to contingencies in the spot market due to transmission and generation outages as well as to demand uncertainties. Our model also accounts for network congestion which is represented through a capacity constrained electricity grid.

We present our formulation as a stochastic equilibrium problem with equilibrium constraints (EPEC) where each generation firm solves a stochastic mathematical program with equilibrium constraints (MPEC, see [13]). Related economic results of the price caps will be analyzed based on a stylized Belgian two-settlement system. The main purpose of this paper is to present the formulation and in particular, to explore the impact of price caps (on both spot and forward prices) in two-settlement markets. In addition, we discuss the details of how to incorporate such price caps in the mathematical formulation while maintaining differentiability of the objective function. The algorithmic details of our new computational approach employed in this paper are developed in a sequel paper. There we introduce an efficient algorithm to find an equilibrium point which is based on the observation that the problem can be reduced to a series of parametric linear complementarity problems.

The remainder of this paper is organized as follows. Related electricity market models are reviewed in the next section, section 3 presents the formulation for the two-settlement market. In section 4, we investigate the implications of hypothetical price caps in a stylized version of the Belgian electricity system. Some remarks are drawn to conclude this paper.

\section{Related Research}

In this section, we will review models with forward contracts and models of spot markets with Cournot competition or transmission constraints. Most spot market models with transmission constraints assume the Cournot conjectural variation. Moreover, if the main purpose is to examine generators' behaviors in the energy market, assuming the agents act as price takers in the transmission market allows the models to formulated as complementarity problems or variational inequalities.

Wei and Smeers [18] consider a Cournot model with regulated transmission prices. They solve variational inequalities to determine unique long-run equilibria in their model. In subsequent work, Smeers and Wei [17] consider a separated energy and transmission market, in which the system operator conducts a transmission capacity auction with power market participants purchasing transmission contracts to support bilateral transactions. They conclude that such a market converges to the optimal dispatch with a large number of market participants.

Hobbs [11] calculates a Cournot equilibrium under the assumption of linear demand and cost functions, which leads to a mixed linear complementarity problem. In a market without arbi- 
trageurs, non-cost based price differences can arise because the bilateral nature of the transactions gives firms more degrees of freedom to discriminate between electricity demands at various nodes. Such result is similar to that in a separated market in [17]. In the market with arbitrageurs, any non-cost differences are eliminated by speculators who buy and sell electricity at nodal prices. This equilibrium is shown to be equivalent to a Nash-Cournot equilibrium in a POOLCO-type market. Hobbs, Metzler and Pang [10] present an oligopolistic market where each firm submits a linear supply function to the Independent System Operator (ISO). They assume that the firms can only manipulate the intercepts of the supply functions, but not the slopes, while the power flows and the pricing strategies are constrained by the ISO's linearized optimal power flow. Each firm in this model faces an MPEC problem with the spatial price equilibrium as the inner problem.

Work on forward markets has focused on the welfare enhancing properties of forward contracts and the commitment value. The basic model in [1] assumes that producers meet in a two-period market where there exists some demand uncertainty in the second period. Allaz shows that the generators have a strategic incentive to contract forward if other producers do not. This result can be understood using the concepts of strategic substitutes and complements of Bulow, Geneakoplos and Klemperer [3]. The availability of the forward market makes a particular producer more aggressive in the spot market. Due to the strategic substitute effect, this produces a negative effect on its competitors' production. The producer having access to the forward market can therefore use its forward commitment to improve its profitability to the detriment of its competitors. Allaz shows, however, that with all producers having access to the forward market, it leads to a prisoners' dilemma type of effect, reducing the profits for all producers. Allaz and Vila [2] extend this result to the case with more than one time period for forward trading to take place. For a case without uncertainty, they establish that as the number of periods, when forward trading takes place, increases to infinity, the producers lose their ability to raise energy prices above their marginal cost.

von der Fehr and Harbord [7] and Powell [16] study contracts and their impact on an imperfectly competitive electricity spot market: the UK pool. von der Fehr and Harbord [7] focus on price competition in the spot market with capacity constraints and multiple demand scenarios. They find that the contracts tend to put downward pressure on spot prices. Although, this provides disincentive to the generators to offer such contracts, there is a countervailing force in which selling a large number of contracts drives a firm to be more aggressive in the spot market, and ensures full dispatch in more demand scenarios. Powell [16] models explicitly re-contracting from Regional Electricity Companies (RECs) after the maturation of the initial portfolio of contracts which is set up after deregulation. He adds risk aversion on the part of RECs to the earlier models. Generators act as price setters in the contract market. He shows that the degree of coordination has an impact on the hedge cover demanded by the RECs, and points to a "free rider" problem which leads to a lower hedge cover chosen by the RECs.

Newbery [14] analyzes the role of contracts as a barrier to entry in the England and Wales electricity market. He extends the earlier work by modelling supply function equilibria (SFE) in the spot market. He further shows that if entrants can sign base load contracts and incumbents have enough capacity, the incumbent can sell enough contracts to drive the spot price below the entrydeterring level, resulting in more volatile spot prices if the producers coordinate on the highest profit supply function equilibrium. Capacity limit however may imply that incumbents cannot play a low enough SFE in the spot market and hence cannot deter entry. Green [9] extends Newbery's model showing that when generators compete in SFE in the spot market, together with the assumption of Cournot conjectural variations in the forward market, no contracting will take place unless the buyers are risk averse and willing to provide a hedge premium in the forward market. He shows that forward sales can deter excess entry, and increase economic efficiency and long-run profits of a large incumbent firm faced with potential entrants. 
Kamat and Oren [12] analyze the welfare and distributional properties of a two-settlement market, which consists of a nodal spot market over 2-node and 3-node networks with a single energy forward market. The system is subject to congestion with uncertain transmission capacities in the spot market, and to generators' market power. Yao, Oren and Adler [19] extend Kamat and Oren's model to more realistic multi-node and multi-zone systems and considers the uncertainty in transmission capacity, generation availability and demand in the spot market. The Cournot equilibrium is modeled as an EPEC in which each generation firm solves an MPEC problem. The model is applied to a six-bus illustrative example, and it is shown that, like the simple cases, the firms have incentives for forward contracting, and that the present of the forward market increases social welfare, and decreases spot price magnitudes and volatilities.

\section{The Model}

We introduce a generic model of the two-settlement electricity system with both spot and forward price caps. The system with either spot or forward price caps can be treated as a special case of this generic model by relaxing the forward or spot price caps.

In this generic model, we model the two-settlement electricity markets as a complete-information two-period game with the forward market being settled in the first period, and the spot market being settled in the second period. The equilibrium is a sub-game perfect Nash equilibrium (SPNE, see $[8])$.

In the forward market, the generation firms determine their forward commitments in order to maximize their expected utility in the spot market while anticipating other firms' forward quantities and the spot market outcomes. The spot market is a subgame with two stages: in stage one, Nature picks the state of the world realizing the actual capacities of the generation facilities and flow capacities of the transmission lines as well as the shape of the demand functions at all nodes. In stage two, firms determine generation quantities to compete in a Nash-Cournot manner while the System Operator (SO) determines how to re-dispatch electricity within the network so as to maximize total social surplus subject to the transmission constraints. Note that the generation firms and the SO take into consideration each other's actions, their decisions are treated as simultaneous moves in our model.

From a mathematical perspective, the model is formulated as an EPEC defining an equilibrium solution among MPECs that characterize the problems of the different competing firms. Each MPEC has two levels where the upper level is a profit maximization problem of the firm in the forward market taking into consideration the spot market equilibrium defined by the lower level problem. This spot market equilibrium is characterized by the set of first order optimality conditions of the firms and the S), where each firm selects its output level so as to maximize spot net revenue while the SO redispatches electricity so as to maximize social welfare subject to the transmission constraints.

\subsection{Notations}

We consider the sets of nodes, transmission lines, zones, generation firms and their generation facilities, as well as the states of world in the spot market.

- $N$ : The set of nodes

- $Z$ : The set of zones. We denote by $z(i)$ as the zone where node $i \in N$ resides.

- $L$ : The set of available transmission lines. 


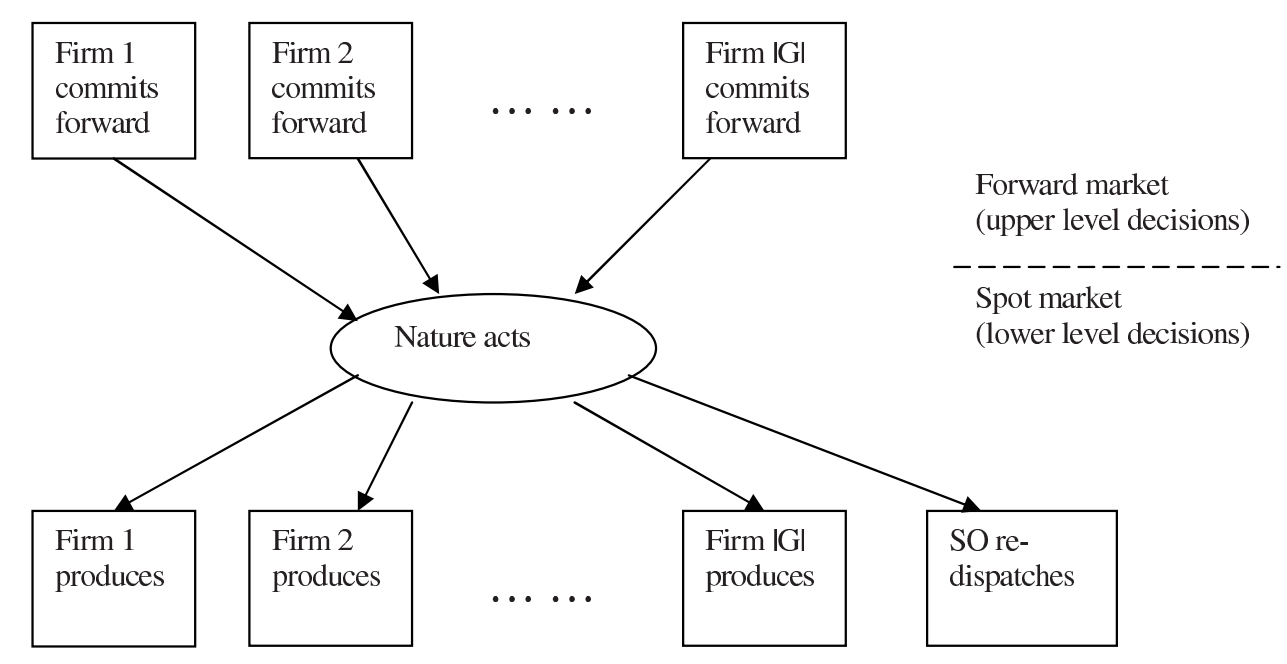

Figure 1: Decision hierarchy

- $C$ : The finite set of possible states of nature in the spot market.

- $G$ : The set of generation firms. $N_{g}$ denotes the set of nodes at which firm $g \in G$ owns generation facilities.

The decision variables related to the forward markets are:

- $x_{g, z}$ : The forward commitment from firm $g \in G$ to zone $z \in Z$.

The decision variables related to the spot markets are:

- $q_{i}^{c}$ : Spot quantity generated at node $i \in N$ in state $c \in C$.

- $r_{i}^{c}$ : Import/export quantity at node $i \in N$ by the $\mathrm{SO}$ in state $c \in C$.

The following exogenous parameters are considered in our formulation:

- $\underline{q}_{i}^{c}, \bar{q}_{i}^{c}$ : The lower and upper capacity bounds of generation facility at node $i \in N$ in state $c \in C$.

- $\bar{u}$ : The spot price cap.

- $\bar{h}$ : The forward price cap.

- $p_{i}^{c}(\cdot)$ : The inverse demand function (IDF) at node $i \in N$ in state $c \in C$. We denote by $\bar{p}^{c}$ the common price intercept across all nodes in each state $c$, and $b_{i}$ as the slopes:

$$
p_{i}^{c}(q)=\bar{p}^{c}-b_{i} q \quad i \in N, c \in C
$$

We assume that the demand curve at each node shifts inwards or outwards in different states, but its slope does not change.

- $C_{i}(\cdot)$ : The generation cost at each node $i \in N$. We assume that the cost function is quadratic convex:

$$
C_{i}(q)=d_{i} q+\frac{1}{2} s_{i} q^{2}
$$


- $K_{l}^{c}$ : The flow capacity limit of line $l \in L$ in state $c \in C$.

- $D_{l, i}^{c}$ : The power transfer distribution factor (PTDF) in state $c \in C$ on line $l \in L$ with respect to node $i \in N$.

- $\operatorname{Pr}(c)$ : The probability of state $c \in C$ in the spot market.

- $\delta_{i}$ : The non-negative weight for node $i \in N$ used to define the spot zonal prices. For each zone $z \in Z$, it holds that $\sum_{i: z(i)=z} \delta_{i}=1$.

\subsection{Prices and Price Caps}

For the sake of generality, we allow different levels of granularity in the financial settlements, with equal granularity being a special case. This is motivated by the fact that, in real markets, we observe different granularity levels in the spot and long-term forward markets - for example in PJM, the western hub representing the weighted average price over nearly 100 nodes is the most liquid forward market. Specifically, the network underlying the nodal spot market is partitioned into a set of zones, each of which contains a cluster of nodes. This suggests three locational prices: spot nodal prices, spot zonal prices (used to settle zonal forward contracts) and forward (zonal) prices.

Spot nodal prices $\hat{p}_{i}^{c}$ are the prices at which the loads are settled at their respective nodes. In each state $c \in C$ of the spot market, the consumption at node $i \in N$ is the sum of the quantity generated by the generator located at that node $\left(q_{i}^{c}\right)$, and the (export or import) adjustment $\left(r_{i}^{c}\right)$ made by the SO. Because the electricity is not economically storable, loads are restricted to be negative, i.e.

$$
q_{i}^{c}+r_{i}^{c} \geq 0, \quad i \in N .
$$

Based on the IDFs, the nodal consumption $q_{i}^{c}+r_{i}^{c}$ is priced at $p_{i}^{c}\left(q_{i}^{c}+r_{i}^{c}\right)$. Actual nodal prices whenever caps are given are expressed as

$$
\hat{p}_{i}^{c}=\min \left\{\bar{u}, p_{i}^{c}\left(q_{i}^{c}+r_{i}^{c}\right)\right\}, \quad i \in N .
$$

The spot zonal price $u_{z}^{c}$ of a zone $z \in Z$ in a state $c \in C$ is used to financially settle zonal forward contracts. It is defined as the weighted average of the nodal prices in the zone with predetermined weights $\delta_{i}$ for the nodes in the zone. These weights are assumed to be given constants in our model (typically these weights reflect the historical load ratios). In mathematical terms, the zonal spot price is given by:

$$
u_{z}^{c}=\sum_{i: z(i)=z} \delta_{i} \hat{p}_{i}^{c}, \quad z \in Z
$$

The forward zonal prices $h_{z}$ are the prices at which forward commitments are agreed upon in the respective zones $z \in Z$. We assume that in equilibrium no profitable arbitrage is possible between the forward and spot zonal prices. This implies that the forward zonal price is equal to the expected spot zonal price; that is,

$$
h_{z}=\sum_{c \in C} \operatorname{Pr}(c) u_{z}^{c}, \quad z \in Z .
$$

With a forward price cap, an upper bound is imposed on these forward prices:

$$
h_{z} \leq \bar{h}, \quad z \in Z \text {. }
$$

In a long-run equilibrium, this upper bound on forward prices typically reflects a backstop price at which new entry becomes ex ante profitable. 


\subsection{The Spot Market and the Lower Level Problem}

We shall now be ready to derive our model. To facilitate the representation, we start from the spot market formulation.

\subsubsection{The Generic Spot Model}

In each state $c \in C$ of the spot market, a generation firm $g \in G$ determines the output of its generation units $q_{i}^{c}\left(i \in N_{g}\right)$. These outputs are restricted within the range determined by the minimal and maximal possible outputs of the plants in that state:

$$
\underline{q}_{i}^{c} \leq q_{i}^{c} \leq \bar{q}_{i}^{c} \quad i \in N_{g}
$$

To avoid computational intractability due to discontinuity in the firms' best-response functions, we follow the common practice in the literature (see [11]) by assuming that the firms ignore the impact of their production decisions on the congestion charges and the settlement of transmission rights. Each firm $g \in G$ receives the revenue of the quantities paid at the spot nodal prices and the financial settlement of its forward contracts settled at the spot zonal prices. In addition to its revenue from the froward contract settlements, firm $g$ 's spot market profit $\pi_{g}^{c}$ is given by:

$$
\pi_{g}^{c}=\sum_{i \in N_{g}} q_{i}^{c} \hat{p}_{i}^{c}-\sum_{z \in Z} u_{z}^{c} x_{g, z}-\sum_{i \in N_{g}} C_{i}\left(q_{i}^{c}\right) .
$$

Each firm $g$ thus solves the following program in each state $c$ of the spot market:

$$
\begin{aligned}
& \max _{q_{i}^{c}} \pi_{g}^{c} \\
& \text { subject to: } \\
& \pi_{g}^{c}=\sum_{i \in N_{g}} q_{i}^{c} \hat{p}_{i}^{c}-\sum_{z \in Z} u_{z}^{c} x_{g, z}-\sum_{i \in N_{g}} C_{i}\left(q_{i}^{c}\right) . \\
& \hat{p}_{i}^{c}=\min \left\{\bar{u}, p_{i}^{c}\left(q_{i}^{c}+r_{i}^{c}\right)\right\}, \quad i \in N_{g} \\
& u_{z}^{c}=\sum_{i: z(i)=z} \delta_{i} \hat{p}_{i}^{c}, \quad z \in Z \\
& h_{z}=\sum_{c \in C} \operatorname{Pr}(c) u_{z}^{c}, \quad z \in Z \\
& h_{z} \leq \bar{h} \quad z \in Z \\
& \underline{q}_{i}^{c} \leq q_{i}^{c} \leq \bar{q}_{i}^{c}, \quad i \in N_{g} \\
& q_{i}^{c}+r_{i}^{c} \geq 0, \quad i \in N_{g}
\end{aligned}
$$

Given the production decisions by the generators, the SO determines for each state $c$ in the spot market the adjustment $r_{i}^{c}$ at each node $i \in N$ (i.e. the import and export quantities at each node). These adjustments are subject to the network thermal constraints on the power flows. We model the electricity flows on the transmission lines in term of the Power Transfer Distribution Factors (PTDF) based on a Direct Current (DC) approximation of the Kirchhoff's law (see [5]). The PTDF $D_{l, i}$ is the proportion of a flow on a particular line $l$ resulting from an injection of one unit of energy at a particular node $i$ and a corresponding one-unit withdrawal at a reference "slack bus". The network feasibility constraints are

$$
-K_{l}^{c} \leq \sum_{i \in N} D_{l, i}^{c} r_{i}^{c} \leq K_{l}^{c}, \quad l \in L
$$


The SO also maintains the real time balance of loads and generation, that is

$$
\sum_{i \in N} r_{i}^{c}=0
$$

The SO's objective is to maximize the social welfare which is defined as the total area under the IDFs minus the total generation cost. Hence, the SO problem in each state $c \in C$ of the spot market is:

$$
\max _{r_{i}^{c}} \sum_{i \in N}\left[\int_{0}^{r_{i}^{c}+q_{i}^{c}} p_{i}^{c}\left(\tau_{i}\right) d \tau_{i}-C_{i}\left(q_{i}^{c}\right)\right]
$$

subject to:

$$
\begin{aligned}
& r_{i}^{c}+q_{i}^{c} \geq 0, \quad i \in N \\
& \sum_{i \in N} r_{i}^{c}=0 \\
& -K_{l}^{c} \leq \sum_{i \in N} D_{l, i}^{c} r_{i}^{c} \leq K_{l}^{c}, \quad l \in L
\end{aligned}
$$

Because the firms' decision variables $q_{i}^{c}$ are treated as given constants in the SO's decision program, the term $C_{i}\left(q_{i}^{c}\right)$ can be dropped from the SO's objective function without affecting the optimal solution.

\subsubsection{Spot Market Smooth Formulation}

The firms and the SO decision problems in the spot market do not have straightforward explicit optimality conditions due to the non-smooth functions characterizing the spot prices. In this section, we reformulate these problems by removing the "min" operator in the characterization of the capped spot nodal prices. It is accomplished by considering separately two cases.

In the first case, the spot price cap is higher than the price intercepts of the IDFs. The spot price cap is thus not binding. Therefore, the spot market formulation is reduced to a variant of that in [19].

The firms' spot decision problems are:

$$
\begin{aligned}
& \mathcal{G}_{g}^{c}: \max _{q_{i}^{c}} \pi_{g}^{c} \\
& \text { subject to: } \\
& \pi_{g}^{c}=\sum_{i \in N_{g}} p_{i}^{c}\left(q_{i}^{c}+r_{i}^{c}\right) q_{i}^{c}-\sum_{z \in Z} u_{z}^{c} x_{g, z}-\sum_{i \in N_{g}} C_{i}\left(q_{i}^{c}\right) . \\
& u_{z}^{c}=\sum_{i: z(i)=z} \delta_{i} p_{i}^{c}\left(q_{i}^{c}+r_{i}^{c}\right), \quad z \in Z \\
& h_{z}=\sum_{c \in C} \operatorname{Pr}(c) u_{z}^{c}, \quad z \in Z \\
& h_{z} \leq \bar{h} \quad z \in Z \\
& \underline{q}_{i}^{c} \leq q_{i}^{c} \leq \bar{q}_{i}^{c}, \quad i \in N_{g} \\
& q_{i}^{c}+r_{i}^{c} \geq 0, \quad i \in N_{g}
\end{aligned}
$$




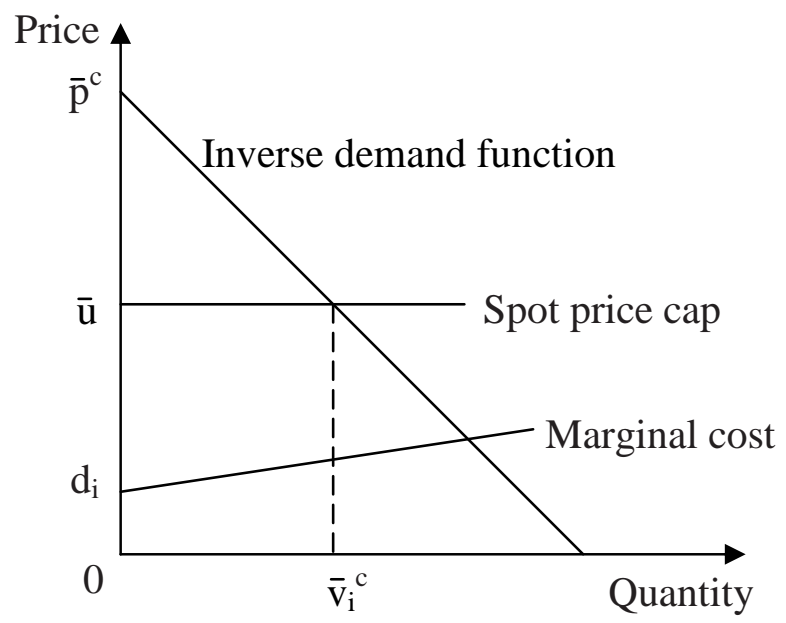

Figure 2: spot price caps

The $\mathrm{SO}$ decision problem is:

$$
\begin{aligned}
\mathcal{S}^{c}: & \max _{r_{i}^{c}} \sum_{i \in N} \int_{0}^{r_{i}^{c}+q_{i}^{c}} p_{i}^{c}\left(\tau_{i}\right) d \tau_{i} \\
& \text { subject to: } \\
& r_{i}^{c}+q_{i}^{c} \geq 0, \quad i \in N \\
& \sum_{i \in N} r_{i}^{c}=0 \\
& -K_{l}^{c} \leq \sum_{i \in N} D_{l, i}^{c} r_{i}^{c} \leq K_{l}^{c}, \quad l \in L
\end{aligned}
$$

When the spot price cap is below the price intercepts of IDFs, it could be binding (see figure 2 ). Note that the standard technique of relaxing the equality $\hat{p}_{i}^{c}=\min \left\{\bar{u}, p_{i}^{c}\left(q_{i}^{c}+r_{i}^{c}\right)\right\}$ with two inequality constraints, $\hat{p}_{i}^{c} \leq \bar{u}$ and $\hat{p}_{i}^{c} \leq p_{i}^{c}\left(q_{i}^{c}+r_{i}^{c}\right)$, may not work here since the sign of the terms containing $\hat{p}_{i}^{c}$ in the firms' objective functions may be positive or negative, depending on the relative magnitude of $x_{g, z}$ and $q_{i}^{c}$. To overcome this complication, we introduce auxiliary variables $v_{i}^{c}$ to represent $\min \left\{q_{i}^{c}+r_{i}^{c}, \bar{v}_{i}^{c}\right\}$, so that

$$
0 \leq v_{i}^{c} \leq q_{i}^{c}+r_{i}^{c}
$$

and

$$
v_{i}^{c} \leq \bar{v}_{i}^{c}=\frac{\bar{p}^{c}-\bar{u}}{b_{i}}
$$

and rewrite the spot nodal prices (considering of the linearity of the demand function) as

$$
\hat{p}_{i}^{c}=\bar{u}-\left(q_{i}^{c}+r_{i}^{c}-v_{i}^{c}\right) b_{i} .
$$

However, writing the spot nodal prices in such a way is correct only if $v_{i}^{c}=\min \left\{q_{i}^{c}+r_{i}^{c}, \bar{v}_{i}^{c}\right\}$. To 


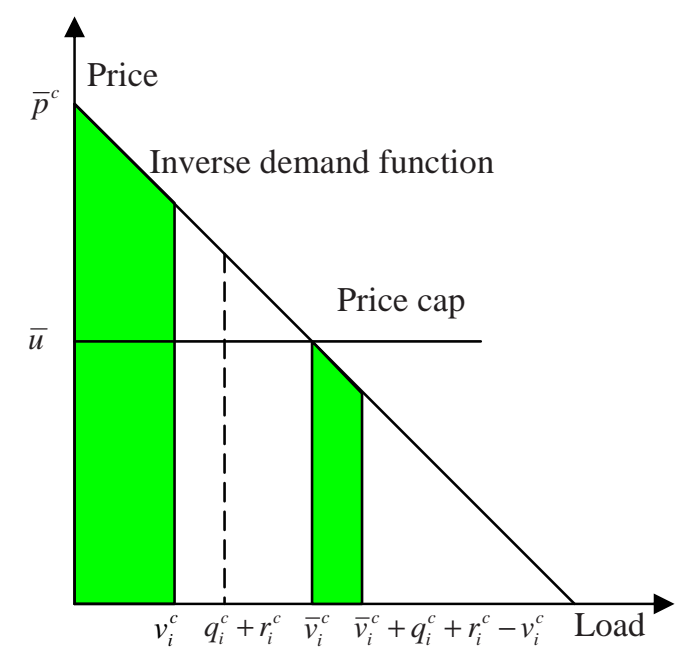

Figure 3: the SO's objective when $q_{i}^{c}+r_{i}^{c} \leq \bar{v}_{i}^{c}$

guarantee this, we reformulate the SO's problem as:

$$
\begin{aligned}
\hat{\mathcal{S}}^{c}: \max _{r_{i}^{c}, v_{i}^{c}} \sum_{i \in N}\left(\int_{0}^{v_{i}^{c}} p_{i}^{c}\left(\tau_{i}\right) d \tau_{i}+\int_{\bar{v}_{i}^{c}}^{\bar{v}_{i}^{c}+r_{i}^{c}+q_{i}^{c}-v_{i}^{c}} p_{i}^{c}\left(\tau_{i}\right) d \tau_{i}\right) \\
\quad \text { subject to: } \\
\quad \sum_{i \in N} r_{i}^{c}=0 \\
\quad \sum_{i \in N} D_{l, i}^{c} r_{i}^{c}, \geq-K_{l}^{c}, \quad l \in L \\
\quad \sum_{i \in N} D_{l, i}^{c} r_{i}^{c} \leq K_{l}^{c}, \quad l \in L \\
\quad v_{i}^{c} \geq 0, \quad i \in N \\
v_{i}^{c} \leq \bar{v}_{i}^{c}, \quad i \in N \\
r_{i}^{c}+q_{i}^{c}-v_{i}^{c} \geq 0, \quad i \in N
\end{aligned}
$$

The two components of the SO's objective function are illustrated in figures 3 and 4 . Notice that, due to the difference of the heights of the two intervals, the maximization of the SO objective function will assign positive load $\left(q_{i}^{c}+r_{i}^{c}-v_{i}^{c}\right)$ in the second interval (beyond $\bar{v}_{i}^{c}$ ) only if the load on the first interval reaches its limit of $\bar{v}_{i}^{c}$. Thus, at the optimal, it holds $v_{i}^{c}=\bar{v}_{i}^{c}$ if $q_{i}^{c}+r_{i}^{c} \geq \bar{v}_{i}^{c}$, and $v_{i}^{c}=q_{i}^{c}+r_{i}^{c}$ if $q_{i}^{c}+r_{i}^{c}<\bar{v}_{i}^{c}$. In another word, the optimal solution will set $v_{i}^{c}$ to $\min \left\{\bar{v}_{i}^{c}, q_{i}^{c}+r_{i}^{c}\right\}$. 


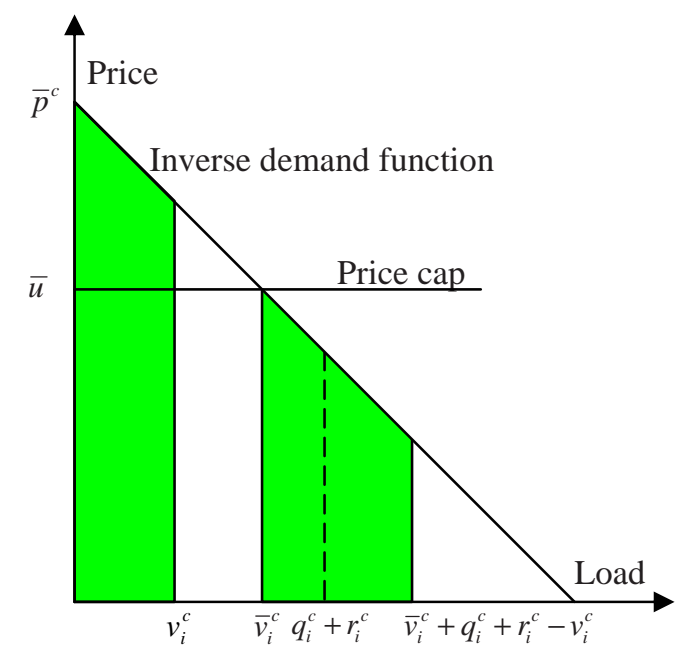

Figure 4: the SO's objective when $q_{i}^{c}+r_{i}^{c}>\bar{v}_{i}^{c}$

The generation firms' spot profit maximization programs can now be written as:

$$
\begin{aligned}
& \hat{\mathcal{G}}_{g}^{c}: \max _{q_{i}^{c}} \quad \pi_{g}^{c} \\
& \text { subject to: } \\
& \pi_{g}^{c}=\sum_{i \in N_{g}}\left(\bar{u}-\left(q_{i}^{c}+r_{i}^{c}-v_{i}^{c}\right) b_{i}\right) q_{i}^{c}-\sum_{z \in Z} u_{z}^{c} x_{g, z}-\sum_{i \in N_{g}} C_{i}\left(q_{i}^{c}\right) \\
& u_{z}^{c}=\sum_{i: z(i)=z}\left(\bar{u}-\left(q_{i}^{c}+r_{i}^{c}-v_{i}^{c}\right) b_{i}\right) \delta_{i}, \quad z \in Z \\
& h_{z}=\sum_{c \in C} \operatorname{Pr}(c) u_{z}^{c}, \quad z \in Z \\
& q_{i}^{c}+r_{i}^{c}-v_{i}^{c} \geq 0, \quad i \in N_{g} \\
& h_{z} \leq \bar{h}_{h} \quad z \in Z \\
& q_{i}^{c} \geq \underline{q}_{i}^{c}, \quad i \in N_{g} \\
& q_{i}^{c} \leq \bar{q}_{i}^{c}, \quad i \in N_{g}
\end{aligned}
$$

It is worth a mention that, if $\bar{v}_{i}^{c}$ are set to zero, programs $\hat{\mathcal{G}}_{g}^{c}$ and $\hat{\mathcal{S}}^{c}$ also capture the generation firms' and the SO's programs when the spot price cap is higher than the price intercepts of IDFs. Therefore, problems $\hat{\mathcal{G}}_{g}^{c}$ and $\hat{\mathcal{S}}^{c}$ along with suitable $\bar{v}_{i}^{c}$ for all nodes $i \in N$ and states $c \in C$ fully represent the agents' spot market decision problems.

\subsubsection{Spot Market Equilibrium Outcomes}

To obtain the simultaneous solutions to the firms' and SO's spot market problems $\left(\hat{\mathcal{G}}_{g}^{c}\right.$ and $\left.\hat{\mathcal{S}}^{c}\right)$, we combine the Karush-Kuhn-Tucker (KKT) conditions, into one problem. Since all the problems involved are strictly concave-maximization problems, we are assured that the solution to the KKT conditions provides the global solutions to all the problems at hand.

In the spot market equilibrium, the shadow prices (the Lagrangian multipliers) corresponding to the constraints $(7)$ for each zone $\left(\eta_{z}\right)$, must be equal across all states for each firm. If not, the firms could increase their profit by decreasing their outputs in states with lower shadow prices while 
increasing the outputs in states with higher shadow prices. Moreover, all firms should face equal shadow prices on these constraints in each zone, because these shadow prices reflect the marginal profit of new entry via the forward price caps. Mathematically, single Lagrangian multipliers should be assigned to (7) for individual zones because this constraint is a coupled constraint shared among all firms' spot market problems in all states.

Let $\alpha^{c}, \lambda_{l-}^{c}, \lambda_{l+}^{c}, \beta_{i-}^{c}, \beta_{i+}^{c}, \mu_{i}^{c}, \rho_{i-}^{c}$ and $\rho_{i+}^{c}$ be the Lagrangian multipliers corresponding to constraints (1)-(6) and (8)-(9) respectively, we obtain the following KKT conditions:

$$
\begin{array}{ll}
\sum_{j \in N} r_{j}^{c}=0 & \\
\bar{p}^{c}-b_{i} v_{i}^{c}-\bar{u}+\left(r_{i}^{c}+q_{i}^{c}-v_{i}^{c}\right) b_{i}-\alpha^{c}+\mu_{i}^{c}+\sum_{t \in L}\left(\lambda_{t-}^{c}-\lambda_{t+}^{c}\right) D_{t, i}^{c}=0, & i \in N \\
\bar{u}-\left(r_{i}^{c}+q_{i}^{c}-v_{i}^{c}\right) b_{i}+\beta_{i-}^{c}-\beta_{i+}^{c}-\mu_{i}^{c}=0, & \\
\bar{u}-2 b_{i} q_{i}^{c}-\left(r_{i}^{c}-v_{i}^{c}\right) b_{i}-d_{i}-s_{i} q_{i}^{c}+\mu_{i}^{c} & i \in N \\
\quad+\delta_{i} b_{i} x_{g, z(i)}+\rho_{i-}^{c}-\rho_{i+}^{c}+\operatorname{Pr}(c) \delta_{i} b_{i} \eta_{z}=0, & \\
\lambda_{l-}^{c} \geq 0, \quad \sum_{j \in N} D_{l, i}^{c} r_{j}^{c} \geq-K_{l}^{c}, \quad\left(\sum_{j \in N} D_{l, i}^{c} r_{j}^{c}+K_{l}^{c}\right) \lambda_{l-}^{c}=0, & i \in N \\
\lambda_{l+}^{c} \geq 0, \quad \sum_{j \in N} D_{l, i}^{c} r_{j}^{c} \leq K_{l}^{c}, \quad\left(K_{l}^{c}-\sum_{j \in N} D_{l, i}^{c} r_{j}^{c}\right) \lambda_{l+}^{c}=0, & l \in L \\
\beta_{i-}^{c} \geq 0, \quad v_{i}^{c} \geq 0, \quad v_{i}^{c} \beta_{i-}^{c}=0, & i \in L \\
\beta_{i+}^{c} \geq 0, \quad v_{i}^{c} \leq \beta_{i+}^{c}, \quad v_{i}^{c} \beta_{i+}^{c}=0, & i \in N \\
\mu_{i}^{c} \geq 0, \quad q_{i}^{c}+r_{i}^{c}-v_{i}^{c} \geq 0, \quad\left(q_{i}^{c}+r_{i}^{c}-v_{i}^{c}\right) \mu_{i}^{c}=0, & i \in N \\
\eta_{z} \geq 0, \quad h_{z} \leq \bar{h}, \quad\left(\bar{h}-h_{z}\right) \eta_{z}=0, & i \in N \\
\rho_{i-}^{c} \geq 0, \quad q_{i}^{c} \geq-\underline{q}_{i}^{c}, \quad\left(q_{i}^{c}+\underline{q}_{i}^{c}\right) \rho_{i-}^{c}=0, & z \in Z \\
\rho_{i+}^{c} \geq 0, \quad q_{i}^{c} \leq \bar{q}_{i}^{c}, \quad\left(\bar{q}_{i}^{c}-q_{i}^{c}\right) \rho_{i+}^{c}=0, & i \in N \\
& i \in N
\end{array}
$$

\subsection{The Forward Market and the EPEC Problem}

As was discussed earlier, we assume that the forward contracts are settled financially. Each firm $g \in G$ takes all its competitors' forward quantities as given, and determines its own forward quantities to maximize its expected spot utility. Assuming the firms are risk neutral, their forward objectives are to maximize their revenues from the forward contracts plus their expected profits from the spot market, subject to the spot market KKT conditions which represent the anticipated

equilibrium in the spot market. Thus for firm $g$, its optimization problem in the forward market is 
the following MPEC problem:

$$
\max _{x_{g, z}} \sum_{z \in Z} h_{z} x_{g, z}+\sum_{c \in C} \operatorname{Pr}(c) \pi_{g}^{c}
$$

subject to:

$$
\begin{aligned}
& \times_{z \in Z} x_{g, z} \in X_{g}, \quad z \in Z \\
& \pi_{g}^{c}=\sum_{i \in N_{g}}\left(\bar{u}-\left(q_{i}^{c}+r_{i}^{c}-v_{i}^{c}\right) b_{i}\right) q_{i}^{c}-\sum_{z \in Z} u_{z}^{c} x_{g, z}-\sum_{i \in N_{g}} C_{i}\left(q_{i}^{c}\right) \\
& u_{z}^{c}=\sum_{i: z(i)=z}\left(\bar{u}-\left(q_{i}^{c}+r_{i}^{c}-v_{i}^{c}\right) b_{i}\right) \delta_{i}, \quad z \in Z \\
& h_{z}=\sum_{c \in C} \operatorname{Pr}(c) u_{z}^{c}, \quad z \in Z
\end{aligned}
$$

and the KKT conditions $(10)-(21)$ for $c \in C$

where the predefined set $X_{g}$ consists of the allowable forward positions for firm $g$. Combining the firms' MPEC problems, the equilibrium problem in the two-settlement markets is an EPEC, where the KKT conditions (10)-(21) denote the lower level problem. An equilbrium of such EPEC problem is a tuple of the variables, including all firms' forward and spot decision variables, the SO's redispatch decisions, as well as the aforementioned Lagrangian multiplies, at which each firm's MPEC problem is solved, and no firm wants to unilaterally change its decisions in both the forward and spot markets.

Next, we aggregate some of the variables so that we can simplify the notations for the firms' MPEC programs by presenting the lower level problem in this EPEC as parametric linear complementarity problem (LCP, see [6]). Define

- $x_{g}$ : The vector of each firm $g$ 's forward quantities. $x_{g}=\left[x_{g, z}, \quad z \in Z\right]$.

- $y: y=\left[y^{c}, \quad c \in C\right]$ where $y^{c}$ is the vector of the Lagrangian multipliers for the inequality constraints in the firms' and the SO's decision problems with respect to state $c$ of the spot market.

- $w: w=\left[w^{c}, c \in C\right]$ where $w^{c}$ is the vector of the slack variables for the constraints corresponding to $y^{c}$.

Thus the spot market KKT conditions become the following parametric LCP with respect to $w$ and $y$ with $x_{g}$ being the parameters

$$
\begin{aligned}
& w=a+\sum_{g} A^{g} x_{g}+M y, \\
& y \geq 0, \quad w \geq 0, \quad y^{T} w=0,
\end{aligned}
$$

where $a, A^{g}$, and $M$ are suitable vector and matrices derived from the KKT conditions (We omit here the detailed representations of $a, A^{g}$, and $M$ ).

Now, the firms' objectives in their MPECs can be expressed as functions $f_{g}\left(x_{g}, x_{-g}, y, w\right)$, where $x_{-g}$ denotes forward commitments of all firms with the exception of firm $g$. 


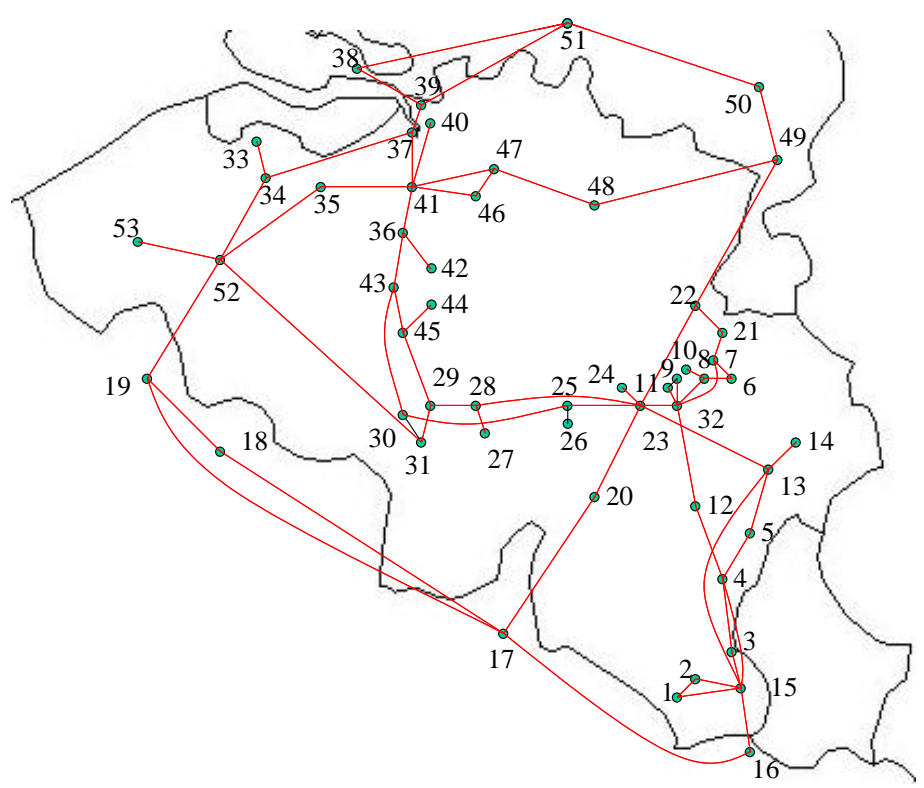

Figure 5: Belgian high voltage network

Using this notation, firm $g$ forward problems is presented as:

$$
\begin{aligned}
& \min _{x_{g}, y, w} f_{g}\left(x_{g}, x_{-g}, y, w\right) \\
& \text { subject to : } \\
& x_{g} \in X_{g} \\
& w=a+A^{-g} x_{-g}+A^{g} x_{g}+M y \\
& y, w \geq 0, \quad y^{T} w=0
\end{aligned}
$$

Here, $x_{g}$ are the decision variables, $y, w$ are the state variables, $x_{-g}$ are the parameters. The EPEC problem in the forward market is hence to find a tuple of $\left(\left\{x_{g}\right\}_{g \in G}, y, w\right)$ at which all firms' MPEC problems are simultaneously solved.

We observe from the programs $\hat{\mathcal{S}}^{c}$ and $\hat{\mathcal{G}}_{g}^{c}$ that $M$ is positive semi-definite and symmetric. Note that since both programs $\hat{\mathcal{S}}^{c}$ and $\hat{\mathcal{G}}_{g}^{c}$ have optimal solutions for all feasible $x_{g}$, the KKT conditions (10)-(21) are solvable as well, and as a result so do the LCP constraints (22)-(23).

To simplify the computation, we assume that the exogenous parameters in our model are perturbed in such a way that the spot decision problems have non-degenerate optimal solutions. Thus, by Theorem 3.1.7 in [6], we are assured that for any $x_{g} \in X_{g}, g \in G$, the LCP constraints (22)-(23) have unique $(y, w)$ solution. The preceding property together with the quadratic objective function $f_{g}\left(x_{g}, x_{-g}, y, w\right)$ allows us to apply an MPEC solution scheme which makes use of the solution uniqueness of the parametric LCP constraints (22)-(23). The details of the solution approach is reported in a sequel paper [21].

\section{The Belgian Electricity Market}

We use a stylized version of the Belgian electricity network to test our model and illustrate the economic results. For completeness of the network, we incorporate some lines in the Netherlands and France. The network has $92380 \mathrm{kV}$ and $220 \mathrm{kv}$ transmission lines, some of which are parallel 
Table 1: Nodal information.

\begin{tabular}{cccc}
\hline $\begin{array}{c}\text { Node } \\
\text { Id }\end{array}$ & $\begin{array}{c}\text { demand } \\
\text { slope }\end{array}$ & $\begin{array}{c}\text { marginal } \\
\text { cost }\end{array}$ & $\begin{array}{c}\text { capa- } \\
\text { city }\end{array}$ \\
\hline 1 & 1 & N/A & 0 \\
2 & 0.82 & N/A & 0 \\
3 & 1.13 & N/A & 0 \\
4 & 1 & N/A & 0 \\
5 & 0.93 & N/A & 0 \\
6 & 0.85 & N/A & 0 \\
7 & 1 & 450 & 70 \\
8 & 1 & N/A & 0 \\
9 & 0.88 & 180 & 460 \\
10 & 0.9 & 180 & $121^{*}$ \\
11 & 1 & 200 & 124 \\
12 & 0.73 & N/A & 0 \\
13 & 1 & N/A & 0 \\
14 & 0.85 & 130 & 1164 \\
15 & 1 & N/A & 0 \\
16 & 1.3 & N/A & 0 \\
17 & 1 & N/A & 0 \\
18 & 0.79 & N/A & 0 \\
19 & 0.68 & N/A & 0 \\
20 & 1.05 & N/A & 0 \\
21 & 1 & N/A & 0 \\
22 & 1.1 & 190 & 602 \\
23 & 1 & N/A & 0 \\
24 & 0.75 & 100 & 2985 \\
25 & 1 & N/A & 0 \\
26 & 0.8 & N/A & 0 \\
27 & 1.13 & N/A & 0 \\
\hline & &
\end{tabular}

\begin{tabular}{|c|c|c|c|}
\hline $\begin{array}{l}\text { Node } \\
\text { Id }\end{array}$ & $\begin{array}{l}\text { demand } \\
\text { slope }\end{array}$ & $\begin{array}{l}\text { marginal } \\
\text { cost }\end{array}$ & $\begin{array}{l}\text { capa- } \\
\text { city }\end{array}$ \\
\hline 28 & 1 & $\mathrm{~N} / \mathrm{A}$ & 0 \\
\hline 29 & 0.93 & $\mathrm{~N} / \mathrm{A}$ & 0 \\
\hline 30 & 0.85 & $\mathrm{~N} / \mathrm{A}$ & 0 \\
\hline 31 & 1 & 180 & 712 \\
\hline 32 & 1 & 580 & 95 \\
\hline 33 & 0.88 & 20 & 496 \\
\hline 34 & 0.5 & $\mathrm{~N} / \mathrm{A}$ & 0 \\
\hline 35 & 1 & 250 & 1053 \\
\hline 36 & 0.73 & $\mathrm{~N} / \mathrm{A}$ & 0 \\
\hline 37 & 1 & 100 & 1399 \\
\hline 38 & 0.85 & $\mathrm{~N} / \mathrm{A}$ & 0 \\
\hline 39 & 1 & $\mathrm{~N} / \mathrm{A}$ & 0 \\
\hline 40 & 1.15 & 100 & 1378 \\
\hline 41 & 1 & 210 & $522^{* *}$ \\
\hline 42 & 0.79 & 180 & 385 \\
\hline 43 & 0.68 & $\mathrm{~N} / \mathrm{A}$ & 0 \\
\hline 44 & 1.03 & 200 & 538 \\
\hline 45 & 1 & $\mathrm{~N} / \mathrm{A}$ & 0 \\
\hline 46 & 1 & $\mathrm{~N} / \mathrm{A}$ & 0 \\
\hline 47 & 1 & 100 & 32 \\
\hline 48 & 0.73 & 220 & 258 \\
\hline 49 & 1.2 & $\mathrm{~N} / \mathrm{A}$ & 0 \\
\hline 50 & 1.5 & $\mathrm{~N} / \mathrm{A}$ & 0 \\
\hline 51 & 1 & $\mathrm{~N} / \mathrm{A}$ & 0 \\
\hline 52 & 1 & $\mathrm{~N} / \mathrm{A}$ & 0 \\
\hline 53 & 1 & 200 & 879 \\
\hline
\end{tabular}

${ }^{*}, * *$ : these numbers are zeros in states 5 and 6 respectively.

N/A: the marginal costs are not applicable to zero capacities.

between the same pair of nodes. For computational purpose, these parallel lines are combined into single lines with adjusted thermal capacities and resistances, the network is hence reduced to 71 transmission lines connecting 53 nodes (see figure 5). 19 nodes in this system have attached generation plants. Insignificant lower voltage lines and small generation plants have been excluded from this example.

The corresponding information of the generation plants is listed in table 1. More details are also given in this table describing the nodal information on the slopes of the inverse demand functions, first-order marginal generation costs, the generation capacities and the historical load ratios (in this example, all marginal generation costs are assumed constant). As to the thermal limits, we ignore the intra-zonal flows and focus only on the flowgates of lines [22,49], [29,45], [30,43], and [31,52]. We assume that there are six states in the spot market. The first state is a state in which the demands are at the shoulder, all generation plants operate at their full capacities, all transmission lines are rated at their full thermal limits. The second state is the same as the first state except that it has on-peak demand. Off-peak state 3 differs from state 1 by the very low demand levels. State 4 denotes the contingency of the transmission line $[31,52]$ being out of service. State 5 and 6 capture the unavailability of two plants at node 10 and 41 respectively. The assumed probabilities 
Table 2: States of the Belgian spot market

\begin{tabular}{ccl}
\hline State & Probability & Type and description \\
\hline 1 & 0.50 & Shoulder state: Demand at shoulder. \\
2 & 0.20 & On-peak state: Demand on the peak. \\
3 & 0.20 & Off-peak state: Demand off-peak. \\
4 & 0.03 & Shoulder demand with line breakdown: Line [31,52] out of service. \\
5 & 0.03 & Shoulder demand with generation outage: Plant at node 10 down. \\
6 & 0.04 & Shoulder demand with generation outage: Plant at node 41 down. \\
\hline
\end{tabular}

of these states are given in table 2. For these states, the price intercepts for the nodal inverse demand functions are set to 500, 1000, 250, 500, 500, and 500 respectively.

We first consider five test cases:

- Case 1: The price-uncapped single-settlement system, i.e. single settlement without (spot) price caps.

- Case 2: The price-capped single-settlement system, i.e. single settlement with (spot) price caps.

- Case 3: The price-uncapped two-settlement system, i.e. two settlements without spot and forward price caps.

- Case 4: The forward-capped two-settlement system, i.e. two settlements with only forward price caps.

- Case 5: The spot-capped two-settlement system, i.e. two settlements with only spot price caps.

Case 1 and 2 are essentially equivalent to a two-settlement system with the allowable forward commitments forced to zeros. In these five cases, we restrict the firms' forward positions to not exceed their total generation capacities in the respective zones.

We first run the test cases with a fixed number of firms and some fixed price caps. We assume that there are two firms competing in the system, and two zones in the network with nodes 1 through 32 being in the first zone and the remaining nodes belong to the second zone. The first firm owns the plants at nodes $7,9,11,31,32,33,35,37,41,47$, and 53 , and the second firm owns the plants at nodes 10,14, 22, 24, 40, 42, 44, and 48. We also assume the spot price cap in cases 2 and 5 is 600 , and the forward price cap in case 4 is 425 .

We find that, when both the spot and the forward prices are not capped, the generation firms have incentives for forward contracting. Moreover, two settlements increase spot generation, decrease the spot prices, and thus increase social welfare as shown in the table 3 . This result is consistent with that in $[1,2,12]$ and $[19]$.

Spot and forward price caps create opposite effects on the firms' incentives for forward commitments. Different incentives further imply different results of spot production and prices as compared to the uncapped two-settlement system. Tables 3 and 4 report the spot generation quantities and spot zonal prices under different cases.

The spot price cap reduces the firms' incentives to commit forward contracts as compared to the uncapped two-settlement system. Specifically, in our simulation, the spot price cap causes firms to commit totally about $90 \%$ of the corresponding forward contract in the uncapped two-settlement 
Table 3: Spot zonal prices

\begin{tabular}{cccccccccccc}
\hline & \multicolumn{2}{c}{ Case 1 } & \multicolumn{2}{c}{ Case 2 } & \multicolumn{2}{c}{ Case 3 } & \multicolumn{2}{c}{ Case 4 } & \multicolumn{2}{c}{ Case 5 } \\
\cline { 2 - 11 } & zone 1 & zone 2 & zone 1 & zone 2 & zone 1 & zone 2 & zone 1 & zone 2 & zone 1 & zone 2 \\
\hline state 1 & 428.29 & 429.04 & 428.29 & 429.04 & 423.95 & 424.79 & 363.30 & 363.03 & 425.27 & 425.60 \\
state 2 & 849.75 & 844.72 & 600.00 & 600.00 & 845.18 & 841.02 & 823.53 & 823.75 & 600.00 & 600.00 \\
state 3 & 232.30 & 232.30 & 232.30 & 232.30 & 224.24 & 224.24 & 189.45 & 189.45 & 229.46 & 229.46 \\
state 4 & 428.20 & 429.14 & 428.20 & 429.14 & 419.75 & 420.97 & 405.46 & 406.32 & 424.38 & 424.56 \\
state 5 & 429.96 & 430.78 & 429.96 & 430.78 & 421.55 & 422.53 & 407.19 & 407.93 & 426.11 & 426.12 \\
state 6 & 431.35 & 432.31 & 431.35 & 432.31 & 423.34 & 424.49 & 409.34 & 410.47 & 428.77 & 428.14 \\
Expected & 473.55 & 473.01 & 423.56 & 424.07 & 468.03 & 467.73 & 425.00 & 425.00 & 421.19 & 421.34 \\
\hline
\end{tabular}

Table 4: Aggregated spot generation

\begin{tabular}{cccccc}
\hline & Case 1 & Case 2 & Case 3 & Case 4 & Case 5 \\
\hline state 1 & 4153 & 4153 & 4440 & 7893 & 4406 \\
state 2 & 8813 & 9694 & 9089 & 10173 & 11014 \\
state 3 & 1049 & 1049 & 1549 & 3500 & 1524 \\
state 4 & 4153 & 4153 & 4667 & 5443 & 4644 \\
state 5 & 4051 & 4051 & 4568 & 5345 & 4545 \\
state 6 & 3971 & 3971 & 4460 & 5211 & 4437 \\
Expected & 4454 & 4630 & 4803 & 7213 & 5164 \\
\hline
\end{tabular}

markets. This observation can be explained in terms of the positive correlation between expected spot output and forward commitments. Spot price caps induce firms to increase production in the on-peak states when the cap is binding and to offset their profit losses by reducing output and increasing prices in the states where the caps are not binding. The net effect is a reduction in expected output across all states and consequently in reduced forward commitments.

Forward price caps, on the other hand, increase the firms' incentives for forward contracting. In our simulation firms' commitments in the forward-capped two-settlement markets are about $80 \%$ higher than those in the same markets without caps. This observation is appealing since it is consistent with the intuition that competitive entry will induce more forward contracting by incumbents in order to deter such entry. Thus, it validates the use of forward caps as a proxy for competitive entry. The more direct explanation is again based on the correlation between expected output and forward commitment. Unlike spot caps that limit prices in on-peak states, forward caps limit expected spot prices across all states via the no arbitrage relationship. Such a limit induces price reduction through an increase in output across all the states resulting in a total increase in expected output and a resulting increase in forward commitment.

We then run more tests to find the sensitivity of forward contracting on the number of firms. We retain the price caps in cases 4 and 5, but vary the number of firms in the system from two to six. We find that the total forward contracting is increasing with the number of firms. This is true for all types of two-settlement systems, no matter whether or not the forward or the spot prices are capped. Figure 6 shows the relationship between the contracting quantity relative to total generation capacity and the number of firms in the market. Again, it is shown that the spot-capped system results in less forward contracting than the uncapped system, which causes less forward contracting than the forward-capped system. The increase in forward contracting with 


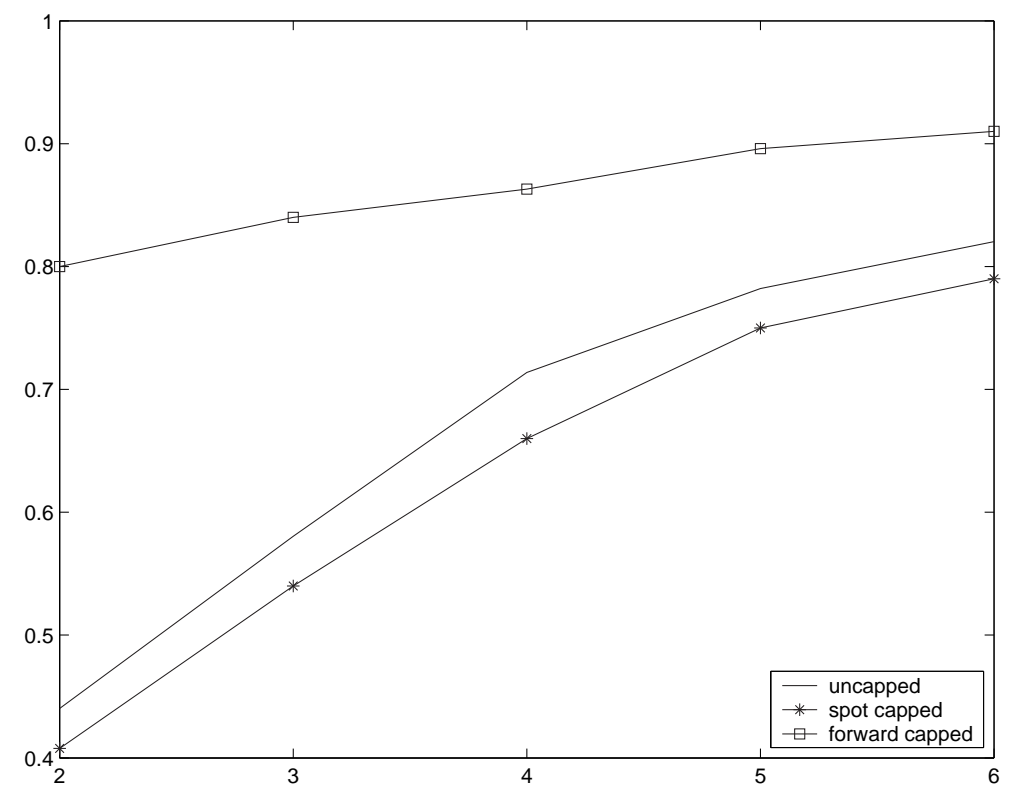

Figure 6: comparison of total forward contracting

the number of firms can be explained by the fact that the prisoners' dilemma effect driving forward contracting incentives is amplified as the number of players increases.

Finally, we investigate the forward contracting at different levels of the spot and forward price caps. Figures 7 and 8 plot the total forward contracting with respect to the price caps and the number of firms. With all other parameters fixed, we find that the lower the spot cap is, the less quantity firms are willing to commit in the forward market. On the other hand, the forward commitments increase as the forward price cap decreases. For any given spot or forward cap, the total forward contracting quantity is increasing in the number of firms.

Note the forward prices are decreasing functions in the forward commitments and the spot generation output quantity, in the case with potential entry the firms will succeed in deterring entry by playing an equilibrium with the forward prices just below the forward cap when they have sufficient capacities and the forward price cap is high enough. However, when the forward price cap is too low or the firms don't have enough capacity, the forward prices will attempt to rise above the forward price cap, and entry to the markets is inevitable as noted by Newbery in [14].

\section{Concluding Remarks}

In this paper, we extend our model presented in [19] to the case in which either the forward prices or the spot prices are capped. We formulate the Cournot equilibrium in the price-capped two-settlement markets as a stochastic equilibrium problem with equilibrium constraints. We also consider the spot market with demand uncertainty and probabilistic contingencies of generation capacity and thermal limits on the transmission lines.

The main goal of this paper is to develop an effective model for analyzing the capping alternatives under a variety of scenarios in the framework of two-settlement markets. We run test cases based on the stylized Belgian electricity market. The resulting equilibrium reveals fewer incentives for the firms to commit forward contracts due to spot price caps, but more incentives for forward contracting under forward price caps induced through competitive entry. However, spot 


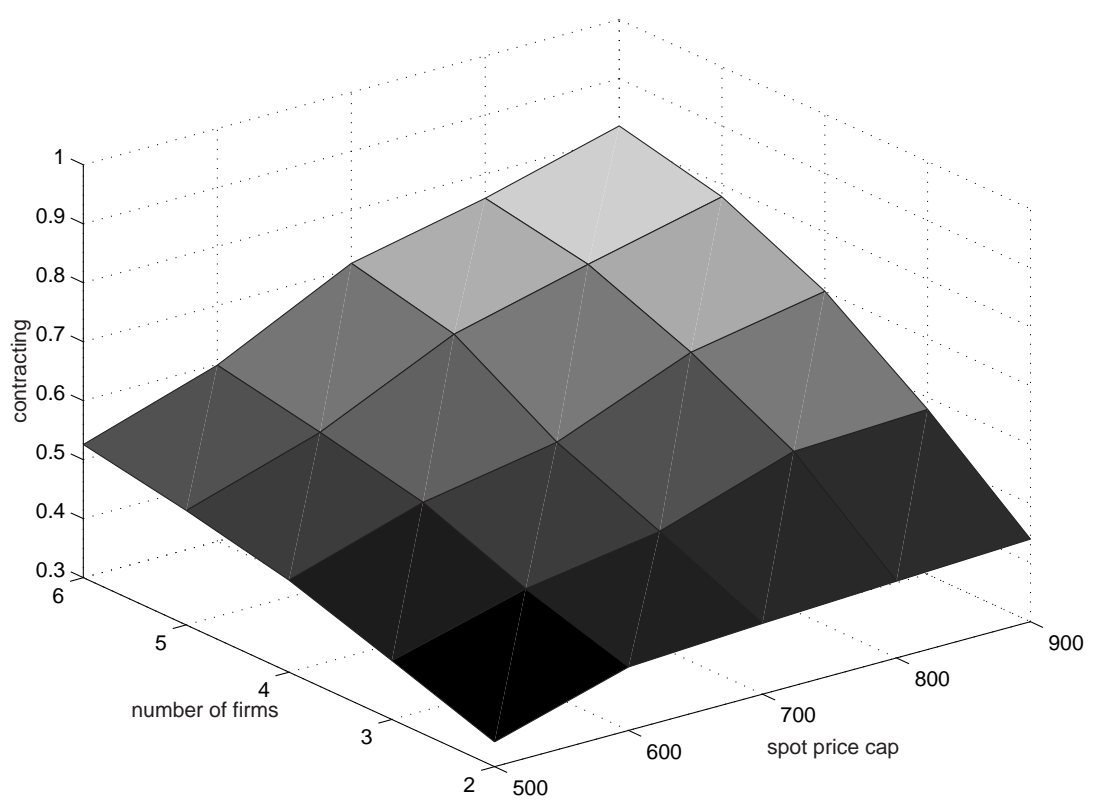

Figure 7: total forward contract with spot caps

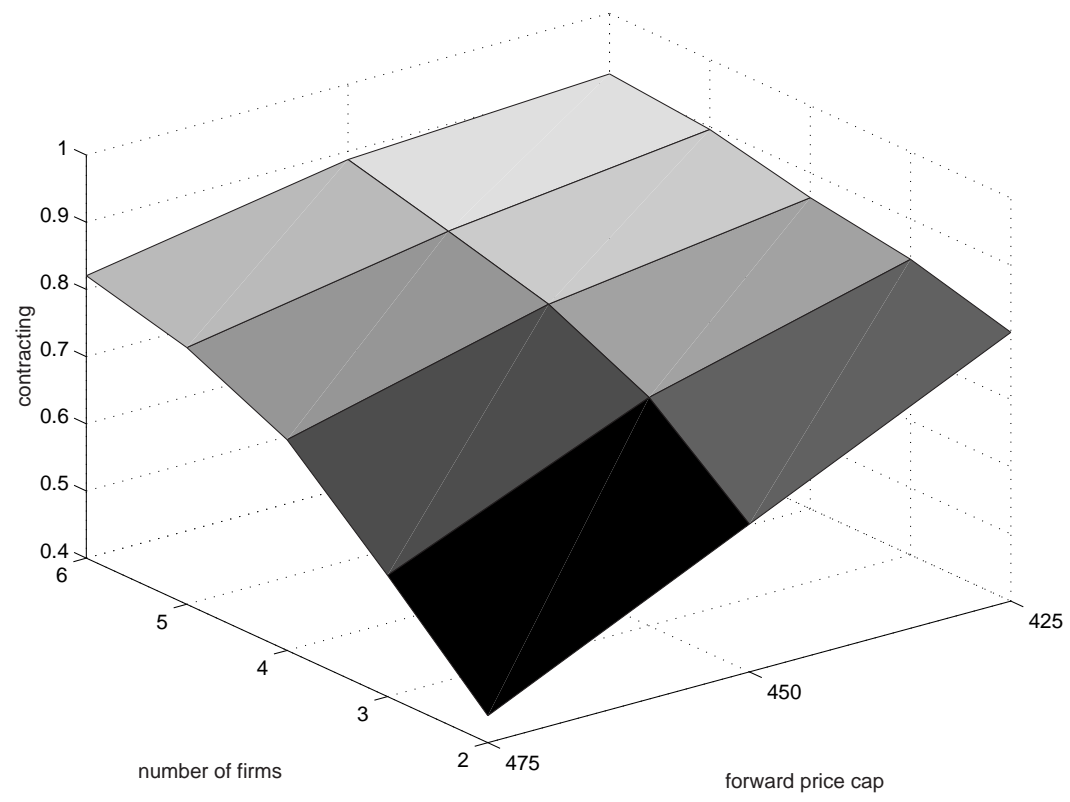

Figure 8: total forward contract with forward caps 
zonal prices, under both cap types, still decrease when forward contracting is possible. Sensitivity studies show that the results are robust and that forward contracting increases as the forward price cap decreases and decreases as the spot cap decreases. We also find that the total level of forward contracting and the sensitivities are amplified as the number of competing firms increases.

Finally, it should be pointed out, that our numerical simulations are limited and their objective was aimed primarily at validating our modelling methodology rather than reaching conclusive economic results which would require far more extensive test studies.

\section{References}

[1] Allaz, B., 1992. Oligopoly, Uncertainty and Strategic Forward Transactions. Internal Journal of Industrial Organization 10, 297-308.

[2] Allaz, B., Vila, J.-L., 1993. Cournot Competition, Forward Markets and Efficiency. Journal of Economic Theory 59, 1-16.

[3] Bulow, J., Geneakolos, J., Klemperer, P., 1985. Multimarket Oligopoly: Strategic Substitutes and Complements. Journal of Political economy 93, 488-511.

[4] Cardell, J., Hitt, C., Hogan, W. W., 1997. Market Power and Strategic Interaction in Electricity Networks. Resource and Energy Economics 19, 109-137.

[5] Chao, H.-P., Peck, S. C., 1996. A market mechanism for electric power transmission. Journal of Regulatory Economics 10(1), 25-60.

[6] Cottle, R. W., Pang, J.S., Stone, R. E., 1992. the Linear Complementarity Problem. Academic Press, Boston, MA, US.

[7] von der Fehr, N.-H. M., Harbord, D., 1992. Long-term Contracts and Imperfectly Competitive Spot Markets: A Study of UK Eletricity Industry. Memorandum no. 14, Department of Economics, Univeristyof Oslo, Oslo, Sweden.

[8] Fudenberg, D., Tirole, J., 1991. Game Theory. the MIT Press, Cambridge, MA, U.S.

[9] Green, R. J., 1999. the Electricity Contract Market in England and Wales. Jounal of Industrial Economics 47(1), 107-124.

[10] Hobbs, B.F., Metzler, C. B., Pang, J.S., 2000. Strategic Gaming Analysis for Electric Power Systems: An MPEC Approach. IEEE Transactions on Power Systems 15(2), 638-645

[11] Hobbs, B.F., 2001. Linear Complementarity Models of Nash-Cournot Competition in Bilateral and POOLCO Power Markets. IEEE Transactions on Power Systems 16(2), 194-202.

[12] Kamat, R., Oren, S. S., 2004. Multi-Settlement Systems for Electricity Markets: Zonal Aggregation under Network Uncertainty and Market Power. Journal of Regulatory Economics 25(1), $5-37$.

[13] Luo, Z.Q., Pang, J.S., Ralph, D., 1996. Mathematical Programs with Equilibrium Constraints. Cambridge University press, Cambridge, MA, US.

[14] Newbery, D. M., 1998. Competition, Contracts, and Entry in the Electricity Spot Market. Rand Journal of Economics 29(4), 726-749. 
[15] Oren, S. S., 1997. Economic Inefficiency of Passive Transmission Rights in Congested Electricity Systems with Competitive Generation. the Energy Journal 18, 63-83.

[16] Powell, A., 1993. Trading Forward in an Imperfect Market: the Case of Electricity in Britain. the Economic Journal 103, 444-453.

[17] Smeers, Y., Wei, J.-Y., 1997. Spatial Oligopolistic Electricity Models with Cournot Firms and Opportunity Cost Transmission Prices. Center for Operations Research and Econometrics, Universite Catholique de Louvain, Louvain-la-newve, Belgium.

[18] Wei, J.-Y., Smeers, Y., 1999. Spatial Oligopolistic Electricity Models with Cournot Firms and Regulated Transmission Prices. Operations Research 47 (1), 102-112.

[19] Yao, J., Oren, S. S., Adler, I., 2004. Computing Cournot Equlibria in Two-settlement Electricity Markets with Transmission Contraints. Proceeding of the 37th Hawaii International Conference on Systems Sciences (HICCS 37). Big Island, Hawaii.

[20] Yao, J., Willems, B., Oren, S. S., Adler I., 2005a. Cournot Equilibrium in Price-capped TwoSettlement Electricity Markets. Proceeding of the 38th Hawaii International Conference on Systems Sciences (HICCS 38). Big Island, Hawaii, January 4-7

[21] Yao, J., Adler, I., Oren, S.S., 2005b. An EPEC algorithm for an Equilibrium Model of Twosettlement Electricity Markets. Manuscript. Department of Industrial Engineering and Operations Research, University of California at Berkeley, Berkeley, California, U.S. 